\title{
Discentes nas aulas online e videoaulas durante a pandemia da Covid-19: um estudo de caso na relação tempo $\mathrm{x}$ dispositivos eletrônicos
}

\author{
Students in online classes and video classes during the Covid-19 pandemic: a case study in the \\ relationship between time and electronic devices
}

Alumnos en clases online y videoclases durante la pandemia Covid-19: un caso de estudio sobre la relación entre el tiempo y los dispositivos electrónicos

\author{
Ederval Pablo Ferreira da Cruz \\ ORCID: https://orcid.org/0000-0002-8545-9990 \\ Universidade Estadual do Norte Fluminense, Brasil \\ E-mail: ederval.cruz@gmail.com \\ Geórgia Regina Rodrigues Gomes \\ ORCID: https://orcid.org/0000-0003-0907-9838 \\ Universidade Estadual do Norte Fluminense, Brasil \\ Universidade Federal Fluminense, Brasil \\ E-mail: georgiagomes@id.uff.br \\ Edson Terra Azevedo Filho \\ ORCID: https://orcid.org/0000-0003-4071-5150 \\ Universidade Estadual do Norte Fluminense, Brasil \\ E-mail: edsonterrafilho@gmail.com
}

\begin{abstract}
Resumo
A pandemia da Covid-19 acelerou a adoção das Tecnologias Digitais de Informação e Comunicação (TDICs) em sala de aula. Porém, diversos problemas ficaram evidentes para os alunos da chamada Geração C, como por exemplo, a fadiga em relação ao acompanhamento das aulas através de telas dos diferentes dispositivos eletrônicos tais como: smartphones, tablets, computadores (Desktop) e notebooks. E essa geração tem como uma das características o consumo de conteúdo no formato de vídeo, porém, de curta duração, objetivos, que busquem solucionar o que procuram, utilizando-se na sua imensa maioria de dispositivos móveis (smartphones e notebooks). Neste trabalho, o foco foi analisar a relação tempo x dispositivos eletrônicos em relação à tolerância no acompanhamento das aulas utilizando de duas metodologias: as videoaulas e aulas online, além de perceber qual é a metodologia que mais os alunos dessa geração se adequaram. Foi realizado um estudo de caso, aplicando-se um questionário aos alunos de diversos cursos e níveis de educação do Instituto Federal do Espírito Santo - Campus Itapina. Os resultados obtidos, que foram estatisticamente tratados, dando maior confiabilidade, mostraram que o tamanho da tela influencia diretamente o tempo de tolerância em acompanhar as aulas sejam em videoaulas ou como aulas online. Percebe-se claramente que existe um gap a ser preenchido no currículo escolar no que tange à adoção de dispositivos móveis e planejamento das aulas utilizando-se desses equipamentos e que levem em consideração as características dos discentes da geração $\mathrm{C}$.
\end{abstract}

Palavras-chave: Geração C; Videoaulas; Covid-19; Aulas online; Ensino.

\begin{abstract}
The Covid-19 pandemic accelerated the adoption of Digital Information and Communication Technologies (TDICs) in the classroom. However, several problems were evident for the students of the so-called Generation $\mathrm{C}$, such as fatigue related to the monitoring of classes through screens of different electronic devices such as: smartphones, tablets, computers (Desktop) and notebooks. And this generation has as one of the characteristics the consumption of content in video format, however, of short duration, objectives, which seek to solve what they are looking for, using mostly mobile devices (smartphones and notebooks). In this work, the focus was to analyze the relationship time $\mathrm{x}$ electronic devices in relation to tolerance in the monitoring of classes using two methodologies: video classes and online classes, in addition to realizing which methodology most students of this generation fit. A case study was carried out, applying a questionnaire to students from different courses and levels of education at the Federal Institute of Espírito Santo - Campus Itapina. The results obtained, which were statistically treated, giving greater reliability, showed that the screen size directly influences the tolerance time in following the classes, whether in video classes or as online classes. It is clearly perceived that there is a gap to be filled in the school curriculum regarding the adoption
\end{abstract}


of mobile devices and class planning using this equipment and that take into account the characteristics of students from Generation C.

Keywords: Generation C; Video classes; Covid-19; Online classes; Teaching.

\begin{abstract}
Resumen
La pandemia de Covid-19 aceleró la adopción de tecnologías de información y comunicación digitales (TDIC) en el aula. Sin embargo, varios problemas fueron evidentes para los estudiantes de la llamada Generación C, como el cansancio en relación al seguimiento de las clases a través de las pantallas de diferentes dispositivos electrónicos como: smartphones, tablets, computadoras (Desktop) y notebooks. Una de las características de esta generación es el consumo de contenidos en formato video, pero de corta duración, objetivos, que buscan resolver lo que buscan, utilizando mayoritariamente dispositivos móviles (smartphones y portátiles). En este trabajo, el enfoque fue analizar la relación tiempo x dispositivos electrónicos en relación a la tolerancia en el seguimiento de clases utilizando dos metodologías: clases de video y clases online, además de darse cuenta de qué metodología encajan la mayoría de los estudiantes de esta generación. Se realizó un estudio de caso, aplicando un cuestionario a estudiantes de diferentes cursos y niveles de educación en el Instituto Federal de Espírito Santo - Campus Itapina. Los resultados obtenidos, que fueron tratados estadísticamente, dando mayor confiabilidad, mostraron que el tamaño de la pantalla influye directamente en el tiempo de tolerancia en el seguimiento de las clases, ya sea en clases de video o como clases en línea. Se percibe claramente que hay un vacío por llenar en el currículo escolar en cuanto a la adopción de dispositivos móviles y la planificación de clases con este equipo y que tenga en cuenta las características de los estudiantes de la Generación C.
\end{abstract}

Palabras clave: Generación C; Clases de video; Covid-19; Clases online; Enseñanza.

\title{
1. Introdução
}

No final de 2019, ainda sendo um pequeno surto, na China iniciava-se a propagação de um vírus que impactaria mais à frente a sociedade global em todas as áreas de forma muito significativa. Rapidamente, tal vírus, apresentado como o novo coronavírus (Covid-19) (Li et al., 2020; Liang et al., 2020), propagou-se pelo mundo, levando à Organização Mundial de Saúde (OMS) a declarar no dia 11/03/2020 que entrávamos em um estágio de pandemia (Cucinotta \& Vanelli, 2020), onde segundo Werneck e Carvalho (2020), na metade de abril/20, poucos meses depois do início na China, já havia ocorrido mais de 2 milhões de casos e 120 mil mortes no por Covid-19.

Contudo, o sistema educacional mundial e, especificamente o brasileiro, que é o foco deste trabalho, não estava preparado para lidar com tal situação, até pelo fato dos profissionais da educação dessa geração nunca terem atravessado extrema situação como a que aconteceu durante o período de pandemia. A sociedade, que vivia uma rotina de "normalidade" se viu tomada por uma doença desconhecida e que nos manteve reféns dentro de nossas casas (Doring, Cruz, \& Ribeiro, 2021).

Assim, graças às diversas Tecnologias Digitais de Informação e Comunicação (TDICs), que permitem a possibilidade de expandir diferentes abordagens na relação ensino e aprendizagem, percebeu-se que, após um período de incertezas e inquietudes trazidos pelo início da pandemia, era possível dar continuidade às aulas, de uma forma diferenciada do ensino tradicional em sala de aula e de outras metodologias que estavam sendo utilizadas. E assim, adota-se o Ensino Remoto, que segundo Moreira e Schlemmer (2020) pode ser compreendido como:

O Ensino Remoto ou Aula Remota se configura então, como uma modalidade de ensino ou aula que pressupõe o distanciamento geográfico de professores e estudantes e vem sendo adotada nos diferentes níveis de ensino, por instituições educacionais no mundo todo, em função das restrições impostas pelo Covid-19, que impossibilita a presença física de estudantes e professores nos espaços geográficos das instituições educacionais.

Muitos foram os desafios enfrentados pelos profissionais da educação na adoção do Ensino Remoto, buscando impactar minimamente o sistema educacional (Joye, Moreira, \& Rocha, 2020). Mas percebeu-se que, os envolvidos dentro de tal sistema não estavam preparados para essa situação pandêmica. Focando especificamente em docentes e discentes, ficou claro que todos foram surpreendidos pela adoção do Ensino Remoto, onde era necessário utilizar das TDICs, nas formas dos 
mais diversos aplicativos e/ou Learning Management Systems (LMS) e/ou de diferentes plataformas como, por exemplo, o Youtube, para o streaming de videoaulas ou o Zoom, Microsoft Teams e Google Meet, para a realização de videoconferências/aulas "ao vivo" (síncronas), que possuem algumas poucas características existentes das aulas presenciais, que mesmo em espaços físicos diferentes, todos os envolvidos estão juntos com a mesma finalidade, dando assim continuidade ao processo de ensino e aprendizagem dos discentes (Nascimento, Mansur, \& Gomes, 2020).

Porém, foi visto que os docentes, de forma geral, não tinham conhecimentos ou não se sentiam confortáveis na utilização das TDICs para criação das aulas, sejam no formato de videoaulas ou na forma de videoconferências. Segundo Klapproth, Federkeil, Heinschke, \& Jungmann (2020), os docentes não estavam preparados para lidarem com os recursos tecnológicos os quais tornaram-se dependentes e assim, percebeu-se que com o passar do tempo, as videoaulas e encontros síncronos via videoconferências, utilizando-se de plataformas já mencionadas, mostravam ser uma estratégia que, se não planejada, sejam de materiais, em relação à duração das aulas, entre outras variáveis, ia reduzindo o engajamento dos alunos, desmotivando-os, tendo como resultado uma participação cada vez menor dos discentes nos encontros síncronos, principalmente.

O mesmo se deu com os alunos, que estavam acostumados com a forma presencial de ensino, onde precisaram se adequar a este novo paradigma de formato de aulas os quais não estavam habituados no seu cotidiano diário. Portanto, os docentes tiveram que se autocapacitarem e os alunos também precisaram se ajustar aos diversos aplicativos/plataformas/LMS utilizados pelas várias instituições de ensino e utilizando-se de diferentes dispositivos com diversos tamanhos de tela, sejam eles smartphones, tablets, computadores de mesa e notebooks.

Não foram somente alunos os afetados por esses problemas. Entre abril/2020 e maio/2020, uma pesquisa realizada pela Microsoft com mais de 2000 profissionais de seis países, mostrou que as longas horas de videoconferência acabou sendo um desafio a ser enfrentado para os colaboradores que trabalhavam no sistema de "home office". A necessidade de ter um nível elevado de concentração para as diversas reuniões, segundo a pesquisa, o cérebro começa a mostrar inícios de fadiga a partir de 30 minutos de videoconferência (Spataro, 2020).

Por isso, não foi discutido de forma profunda, em busca de um real aprendizado e principalmente, sabendo lidar com as novidades enfrentadas pelos discentes no uso de ferramentas que não faziam parte do seu cotidiano, o tempo ideal de duração dessas videoaulas e dos encontros síncronos, e também como a adoção dos diferentes dispositivos eletrônicos, tais como smartphones, notebooks, computadores, tablets, entre outros equipamentos, utilizados como meio para acesso aos conteúdos de estudo impactaram no engajamento dos alunos em acompanharem as videoaulas e videoconferências sem se sentirem fadigados em frente aos diferentes tamanhos de tela. Portanto, o presente trabalho busca, através de um estudo de caso, responder à seguinte pergunta: Em tempos de pandemia, qual foi o impacto na utilização de dispositivos eletrônicos (smartphones/tablets/notebooks e computadores) no tempo de tolerância dos alunos em relação ao acompanhamento de aulas, sejam no formato de videoaulas (aulas gravadas) ou online (aulas em tempo real) durante o período do ensino remoto por causa do distanciamento social da Covid-19?

O presente artigo encontra-se estruturado da seguinte forma. Na Seção 2 é apresentado o referencial teórico que dá base ao presente trabalho. Na Seção 3 é apresentada a metodologia utilizada na pesquisa. Na Seção 4 os resultados obtidos são discutidos e o artigo é finalizado na Seção 5.

\section{Referencial Teórico}

A pandemia da Covid-19 fez com que o processo de ensino e aprendizagem fosse repensado em caráter de urgência. Muitos professores enfrentaram desafios tecnológicos, mudanças pedagógicas e ainda conheceram cada aluno dentro das suas 
limitações de aprendizagem no ensino remoto e socioeconômicas que foram claramente expostas deixando evidente a desigualdade dos alunos em relação ao acesso aos conteúdos (Artacho, Martínez, Martín, Marín, \& Garcia, 2020).

Obviamente, diversos setores não tinham noção do que aconteceria, os impactos e quais seriam as atitudes a serem tomadas. Na educação, obviamente não foi diferente. A Figura 1 mostra que no dia 26/04/2020, quase 90\% dos alunos estavam impossibilitados de terem aulas ao redor do mundo devido à pandemia da Covid-19.

Figura 1 - Monitoramento global de escolas fechadas devido à Covid-19.

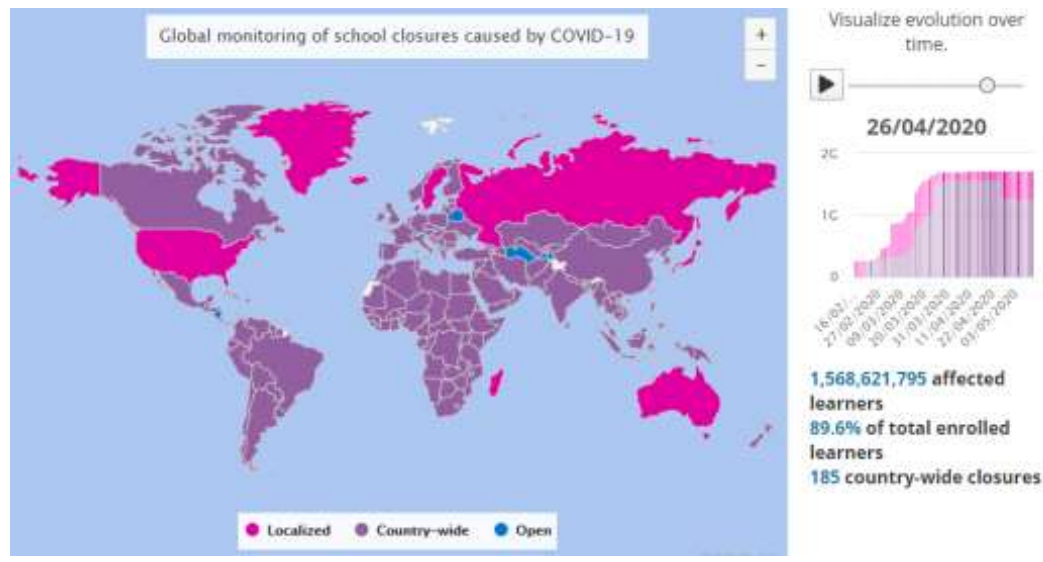

Fonte: https://en.unesco.org/news/covid-19-educational-disruption-and-response.

Para o aluno, o estudar no conforto de casa tem diversas vantagens incluindo economia nos custos de transporte, menos estresse devido aos congestionamentos do trânsito e a possibilidade de ter maior tempo livre (Purwanto, et al., 2020). Lógico que também possui desvantagens como tédio, redução da motivação, custos financeiros maiores com consumo de energia entre outros.

Porém, estudar nessa pandemia mostrou outras características, que são extremamente preocupantes reduzindo fortemente o engajamento do aluno na participação nas aulas online para as aulas síncronas assim como no assistir as videoaulas. Além problemas já apresentados, ainda somam-se a isso a perda de foco devido às inúmeras notificações de aplicativos recebidas no smartphone, ligações recebidas, diversas abas abertas em um browser, baixa interação com outros colegas de sala, entre outros que fazem com que o aluno durante o seu momento de concentração, tenha a mesma quebrada, dificultando o processo de aprendizagem do mesmo.

Segundo o estudo de Peper, Wilson, Martin, Rosegard e Harvey (2021) realizado com 325 estudantes de graduação mostrou que, para a imensa maioria, conforme Figura 2 a aprendizagem ficou mais difícil devido ao fato de adotarem a ferramenta Zoom sem uma preparação prévia dos materiais a serem utilizados especificamente para essa nova forma de aprendizagem e que, com diversas aulas realizadas desta forma, acabaram levando os alunos a sofrerem da chamada "Fadiga do Zoom" (Lee, 2020; Bailenson, 2021) ou "Fadiga de tela" (Peper, 2021). 
Figura 2 - Nível de dificuldade relatado pelos alunos durante a pandemia da Covid-19.

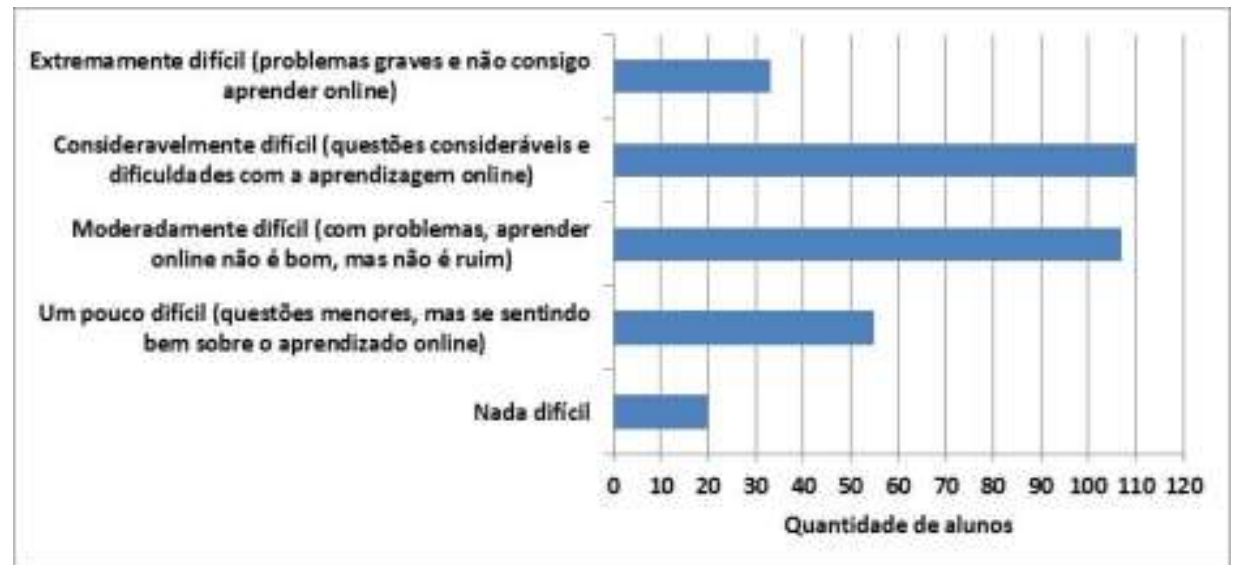

Fonte: Adaptado de Peper (2021).

Além desse fator, outros diversos tais como a falta de dispositivos eletrônicos para acompanhar as aulas, de acesso à internet, falta de motivação, dificuldades de aprendizado (especialmente nas matérias de exatas), problemas psico-emocionais como dores de cabeça, dores de estômago, crises de ansiedade, cansaço mental e visual, dores no pescoço, depressão, entre outras, relatados por diversas pessoas, certamente influenciaram diretamente em perdas significativas de uma aprendizagem efetiva (Intolo et al.,2019; Leeb et al., 2020; McGinty, Presskreischer, Anderson, Han, \& Barry, 2020; Peper, Harvey, \& Faass, 2020).

As videoconferências através dos diversos aplicativos como Zoom, Microsoft Teams, Google Meet e outros, para muitos alunos foi uma novidade, porém, percebeu-se que os mesmos começaram a ficar desgastados com as aulas online, devido, especialmente à duração das mesmas, assim como as diversas videoaulas disponibilizadas para serem assistidas.

O trabalho de Niemi e Kouza (2020) realizou um estudo da percepção de professores e estudantes secundaristas em uma escola na Finlândia durante dois meses no período da pandemia. No artigo uma das conclusões foi que os estudantes reclamaram da grande carga de trabalho imposta pelas atividades assim como também da fadiga, levando os alunos a se sentirem desmotivados.

O impacto no uso excessivo dos smartphones, computadores de mesa e notebooks na visão é avaliado no trabalho de Atmojo, e Nugroho (2020), onde os professores e alunos reclamam do efeito da radiação emitida por esses dispositivos durante longos períodos de tempo. Porém, no trabalho, não deixa claro quais seriam esses períodos de tempo.

A “desordem da visão", proveniente pelo smartphone, é um complexo de problemas relacionados ao olho e visão que são associados ao uso da tela digital e é uma das crescentes preocupações com o bem-estar que apareceram com a chegada dos smartphones e tablets. Especialmente esse problema ficou mais evidente para os estudantes durante a pandemia da Covid-19, devido às aulas online e videoaulas utilizadas pelos professores como metodologia de ensino. No estudo de Hundekari, Sisodiya, e Kot (2021) os autores conduziram uma pesquisa com 280 estudantes de graduação em medicina que tiveram aulas online durante 5 meses. Através da aplicação de um questionário aplicado, os resultados mostraram que os alunos que utilizaram de telas menores, tem maior probabilidade de sofrerem da "desordem da visão". Mas o trabalho não diz, com quanto tempo de uso desses dispositivos os discentes começam a sofrerem de fadiga ocular que podem acarretar ao problema da "desordem da visão".

O tamanho da tela de dispositivos móveis e seu impacto na aprendizagem por vídeo é discutida no trabalho de Maniar, Bennet, Hand, e Allan (2008). Os resultados encontrados mostram que o tamanho da tela pode influenciar no aprendizado dos alunos. Porém, os dispositivos móveis utilizados nessa pesquisa variavam de 1.65 polegadas - 2.75 polegadas, que é 
significativamente menor do que o tamanho das telas dos smartphones atuais. Além disso, estamos atualmente lidando com a Geração C, que segundo o Google Brasil (2012), 91\% dessa geração dormem ao lado do smartphone (que tem como principal característica estar conectada na imensa maioria do tempo e que já lidam com a web, inseridos em comunidades virtuais, games online, plataformas de streaming, entre outras (da Silva, \& Pinto, 2009). Vale destacar também, que em nenhum momento, os pesquisadores se preocuparam em investigar o tempo que o público-alvo pesquisado acha ideal para as aulas gravadas no formato de vídeo.

Já no trabalho de Popescu, Tătuc, e Dobromirescu (2021), os autores buscaram analisar se o bem-estar na educação online é inferior ao da educação clássica durante a pandemia da Covid-19. A pesquisa mostrou que diversos fatores tais como o estado emocional, interação social, materiais de estudos disponibilizados, fadiga ocular, dores de cabeça, entre outros fatores, diretamente causados pela atividade prolongada em frente do computador influenciaram diretamente no desempenho e engajamento dos alunos.

Percebe-se que a adoção das videoaulas e ferramentas de videoconferências durante a pandemia da Covid-19, trouxe à tona diversos problemas que estavam ainda em caráter inicial devido à adoção, ainda em fase inicial, desses recursos, especialmente para os alunos do Ensino Médio e Superior das escolas públicas, que não estavam acostumados, no seu cotidiano com essa abordagem na sua vida escolar, apesar de fazerem parte da Geração C já explicada anteriormente.

\section{Metodologia}

Quando aborda-se metodologia, além de ser discutido o campo teórico do estudo sobre a temática escolhida, a pesquisa também pode ser abrangida, estendida, pois ela permite que novos estudos possam ser propostos a partir deste, e também buscando que novos conhecimentos sejam adquiridos.

Realizou-se um estudo de caso, através da aplicação de um questionário, que segundo Gil (2002, p.116) "consiste basicamente em traduzir os objetivos específicos da pesquisa em itens bem redigidos”, para os alunos dos cursos técnicos integrados ao Ensino Médio e cursos Superiores (Bacharelado em Agronomia, Licenciatura em Ciências Agrícolas e Licenciatura em Pedagogia) do IFES - Campus Itapina, via Google Formulários, enviando a eles um link de acesso para responder. O questionário foi dividido em quatro partes: (1) buscou-se obter informações sobre a idade e qual curso o discente estava cursando durante o período letivo 2020/2021; (2) teve o intuito de obter informações sobre a relação do discente com as videoaulas nos mais diversos dispositivos; (3) objetivou-se buscar informações sobre a relação do discente com as aulas online nos mais diversos dispositivos; (4) finalizou-se buscando saber qual foi a metodologia que mais agradou durante o período 2020/2021. O questionário ficou disponível para ser respondido no período de 03 de maio de 2021 até 17 de maio de 2021, totalizando 15 dias para respostas.

Ao iniciar a pesquisa, os alunos tiveram acesso a um Termo de Consentimento Livre e Esclarecido (TCLE) deixando claro que os dados pessoais não seriam utilizados e expostos. As perguntas eram de ordem fechada, ou seja, alternativas para as respostas, e em algumas perguntas, podendo assinalar mais de uma opção, caso achassem necessário. Depois de fechado o período de respostas ao questionário, tivemos um total de 149 respostas, divididos pelas séries e cursos pesquisados, conforme Figura 3, de uma população total de 1040 alunos possíveis. 
Figura 3 - Series e cursos dos alunos que participaram da pesquisa.

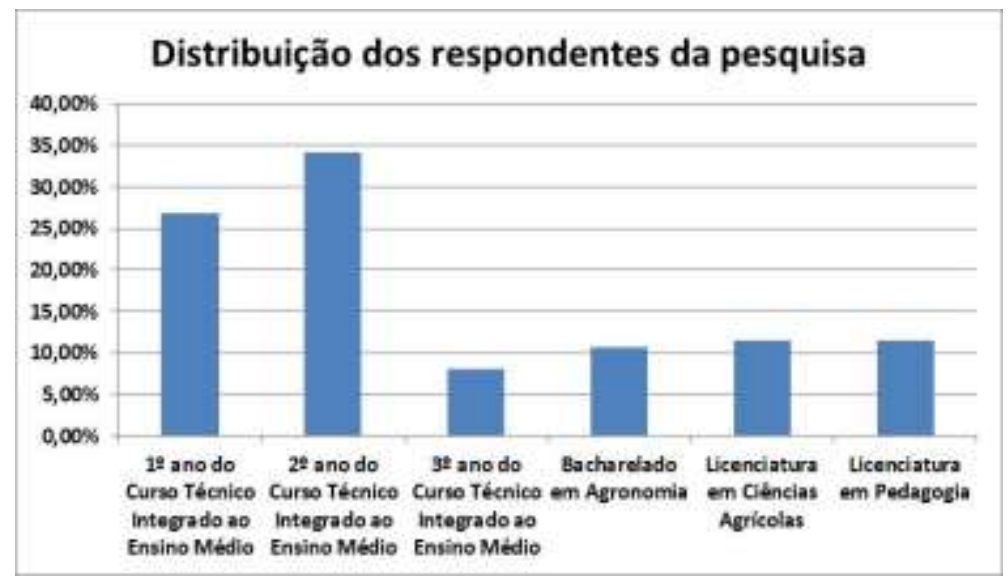

Fonte: Dos próprios autores.

Uma outra pergunta feita foi sobre a idade dos alunos. O objetivo desta pergunta foi analisar qual é a faixa etária predominante dos alunos dentro da escola estudada. Os resultados exibidos na Figura 4, mostraram que a faixa dos 15 a 19 anos é a que tem o maior quantitativo de alunos, cujo público tem como uma característica predominante o uso constante da tecnologia.

Figura 4 - Faixa etária dos alunos pesquisados.

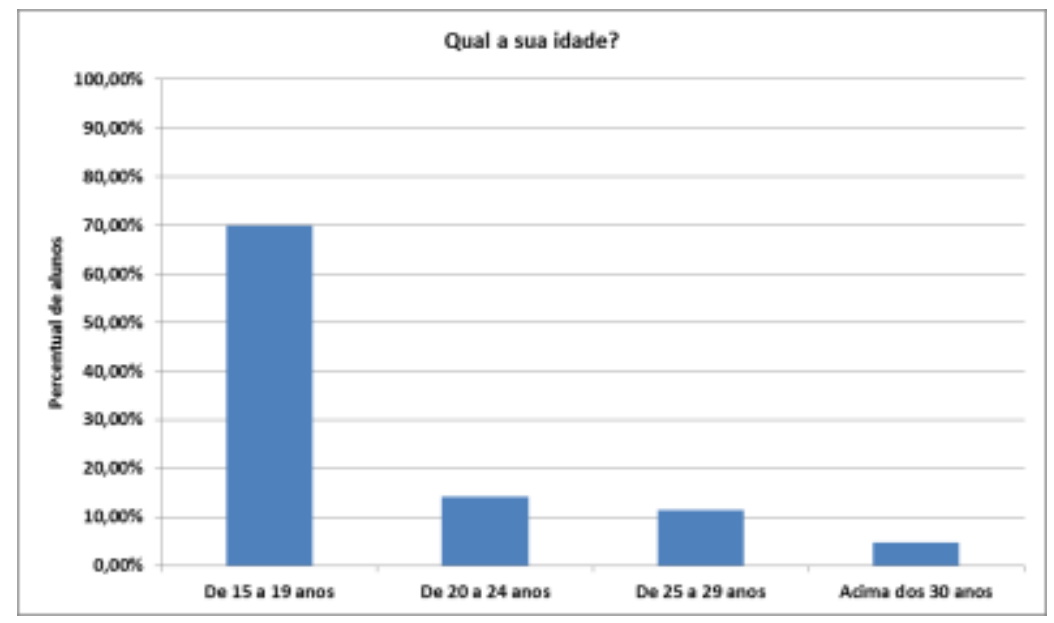

Fonte: Dos próprios autores.

Um dos recursos utilizados para validação dos resultados obtidos é o cálculo da margem de erro, também conhecida como intervalo de confiança, que tem como objetivo indicar em que medida se pode esperar que os resultados da pesquisa fossem um reflexo das opiniões reais da população total. Portanto, a ideia é mostrar, a partir das respostas de um grupo menor (respondentes da pesquisa), o quanto elas podem representar de um grupo maior (população total). Logo, quanto menor a margem de erro, mais confiança tem-se nos resultados. Quanto maior a margem de erro, maior a discrepância entre os resultados e as opiniões da população total.

Para facilitar o entendimento, a margem de erro é um intervalo de valores acima e abaixo dos resultados reais de uma pesquisa. Por exemplo, se 60\% das respostas foram "sim" com uma margem de erro de 5\%, significa que entre 55\% e $65 \%$ da população considera que a resposta é “sim”. E para realizar o cálculo da margem de erro segue-se a seguinte fórmula:

$$
\text { Margem de erro }=z x \frac{\sigma}{\sqrt{n}}
$$


onde n é o tamanho da amostra, $\sigma$ é o desvio padrão e z é o escore-z.

Vale destacar que os resultados apresentados na próxima seção desta investigação, para maior confiabilidade e validade estatística, apresentam um grau de confiança de $95 \%$ e uma margem de erro de $7 \%$.

\section{Resultados e Discussão}

A pesquisa buscou investigar a relação tempo $\mathrm{x}$ dispositivos eletrônicos para os discentes no que tange à adoção de videoaulas e aulas online.

Antes de analisar quais foram os dispositivos eletrônicos e quanto tempo os alunos consideraram toleráveis, isto é, sem se sentirem cansados, fadigados ao acompanharem as aulas usando tais dispositivos, a Figura 5 traz, dentre os respondentes, o percentual de discentes que tiveram acesso às videoaulas e aulas online, que foram adotados pelos professores como metodologias durante o ano letivo 2020/2021 na pandemia. É possível perceber que quase em sua totalidade $(92,62 \%)$ dos alunos utilizaram de videoaulas disponibilizadas pelos professores, que foram gravadas pelos próprios ou que também podem ter sido indicadas como material de apoio. Já 75,84\% dos discentes participaram de aulas online realizadas pelos professores, através dos mais diversos softwares e plataformas disponíveis, como forma de metodologia para a realização de aulas durante o período pandêmico.

Figura 5 - Metodologias adotadas pelos docentes durante a pandemia da Covid-19.

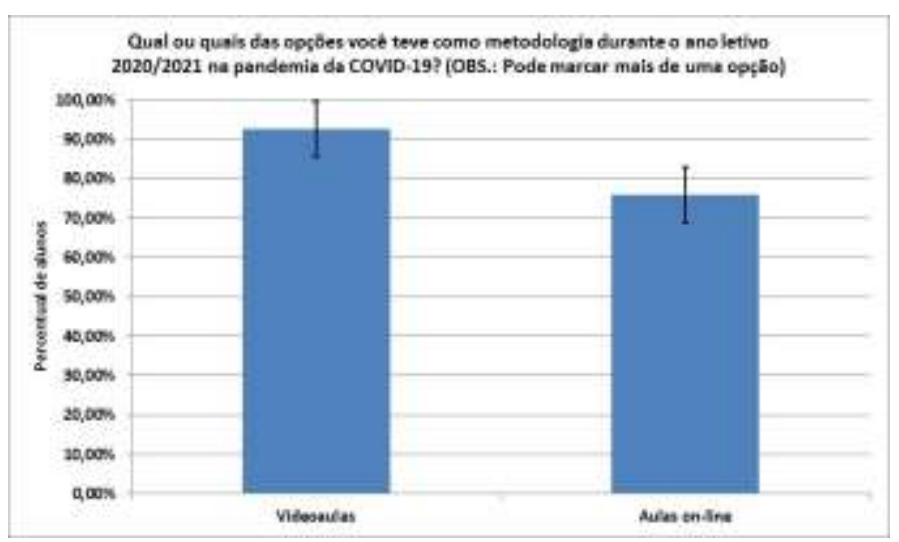

Fonte: Dos próprios autores.

Já na Figura 6, buscou-se analisar qual ou quais foram os dispositivos utilizados para acessar as videoaulas disponibilizadas pelos professores. Os alunos poderiam utilizar mais de um dispositivo. Os resultados obtidos, tanto no acesso às videoaulas (à esquerda) quanto às aulas online (à direita) pelos dispositivos móveis (smartphone e notebook) que graças à facilidade da mobilidade, devido às características desses dispositivos, o aumento do poder de processamento e armazenamento, juntamente com a ampliação no acesso à internet permitindo acesso em locais cada vez mais remotos, são características que deixam clara uma tendência que tem sido vistas nas mais diversas áreas, inclusive na educação, que é a crescente adoção dos dispositivos móveis como recurso para consumo de conteúdo. Vale destacar que, mesmo sendo um dispositivo móvel, o resultado obtido pelo equipamento tablet mostra-se irrelevante, não impactando os demais resultados demonstrados. 
Figura 6 - Dispositivos utilizados pelos discentes para acesso aos conteúdos durante a pandemia da Covid-19.
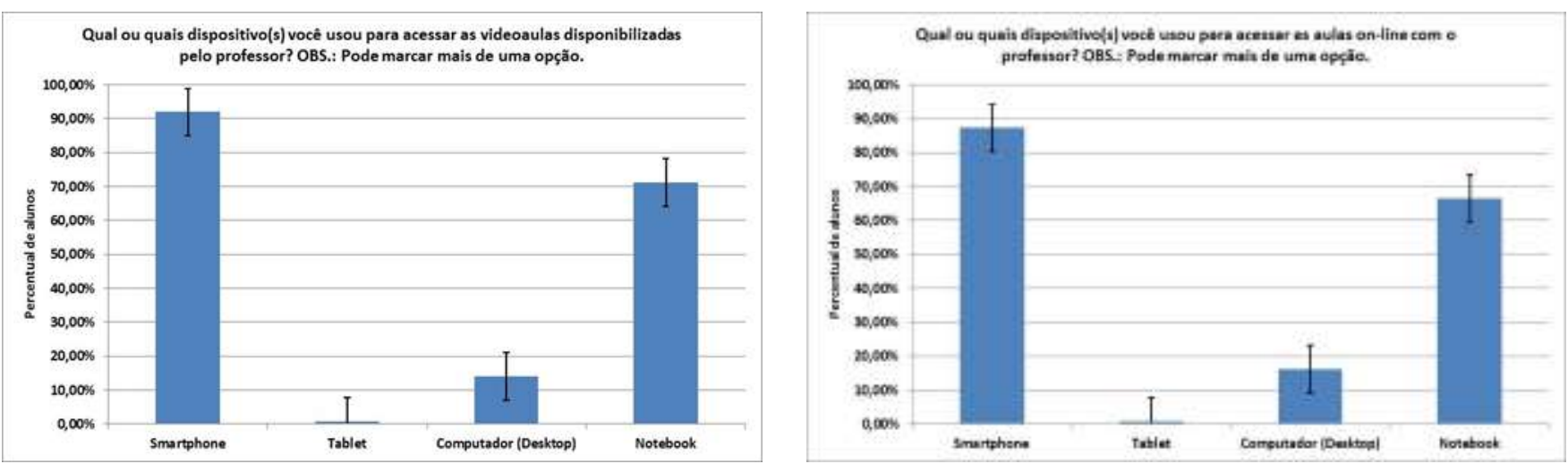

Fonte: Dos próprios autores.

Considerando o smartphone como dispositivo a ser utilizado, foi questionado quanto tempo o discente considera ser tolerável para ver uma videoaula (resultados à esquerda) e uma aula online (resultados à direita). Os resultados, exibidos na Figura 7, mostram que independente da metodologia de aula, a maioria dos aprendizes relataram que até 30 minutos é um tempo aceitável para assistirem as videoaulas (64,23\%) quanto às aulas online (56,53\%). Um dos motivos pelo qual a partir dos 30 minutos o percentual começa a ser menor pode ser relativo ao tamanho reduzido da tela do smartphone, quando comparado com outros dispositivos já relatados na Figura 6, que com o passar do tempo os estudantes começam a se sentir fadigados visualmente e também o nível de foco e atenção que com o passar do tempo vai reduzindo.

Figura 7 - Tempo de tolerância dos discentes durante a pandemia da Covid-19 ao utilizar o smartphone.
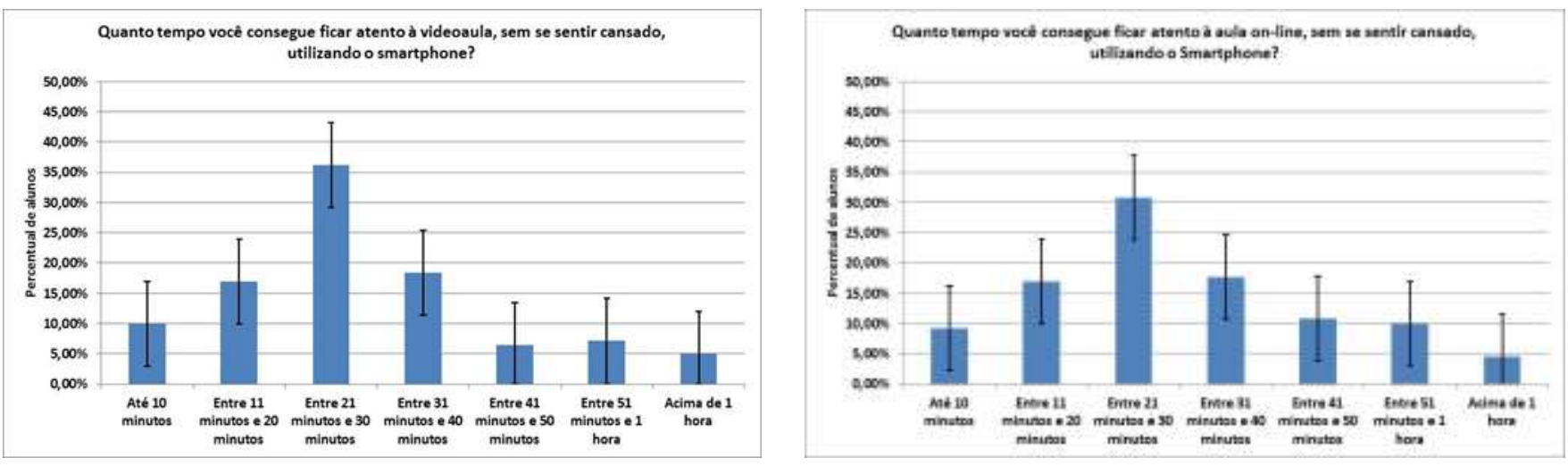

Fonte: Dos próprios autores.

Na Figura 8 a ideia era verificar o nível de tolerância em relação ao acompanhamento das aulas na sua forma de vídeo aulas (resultados à esquerda) e aulas online (resultados à direita) utilizando o notebook como equipamento móvel. O notebook, comparado ao smartphone, possui tamanho de tela maior e poder de processamento e armazenamento superior, porém com menor autonomia em relação à bateria. Os resultados mostram que o fato de ter tela maior do que o smartphone, maior é a tolerância à fadiga visual no acompanhamento das aulas. A maioria $(68,15 \%)$ consegue ter tolerância acima dos 31 minutos ao assistirem videoaulas, assim como 73,34\% conseguem assistir de forma satisfatória as aulas online que venham a durar tempos acima dos 31 minutos. 
Figura 8 - Tempo de tolerância dos discentes durante a pandemia da Covid-19 ao utilizar o notebook.
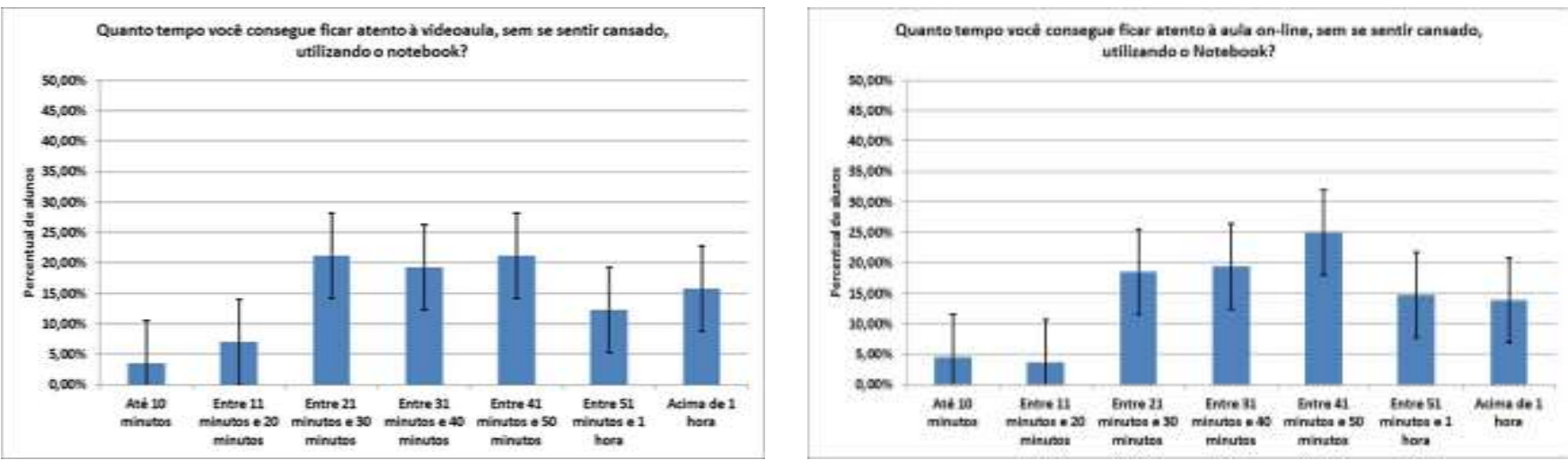

Fonte: Dos próprios autores.

Obviamente, até para uma comparação justa entre dispositivos móveis e não móveis, de acordo com o foco do presente artigo, o computador (Desktop) foi dado como uma das opções para resposta, conforme mostrado na Figura 6 . Assim como nas Figuras 7 e 8, a ideia da Figura 9 era verificar o nível de tolerância em relação ao acompanhamento das aulas na sua forma de videoaulas (resultados à esquerda) e aulas online (resultados à direita), porém utilizando um dispositivo que não possui a característica de mobilidade como o notebook e smartphone, mas que, de forma geral, possui tela maior do que a de um notebook. Os resultados mostram claramente que a tolerância dos discentes para acompanharem as videoaulas aumenta conforme o tamanho da tela, onde 66,66\% afirmam que suportam de forma agradável a metodologia de videoaulas acima de 31 minutos. Vale destacar que, comparativamente com o notebook, é visto que com o uso do computador (Desktop), maior é o percentual de alunos que toleram ver videoaulas acima de 51 minutos. A mesma interpretação vale para os alunos que assistem às aulas online, onde quando comparado os resultados do computador (Desktop) com o notebook, a tolerância para tempos maiores de aulas online (acima de 51 minutos) aumenta.

Figura 9 - Tempo de tolerância dos discentes durante a pandemia da Covid-19 ao utilizar o computador (Desktop).
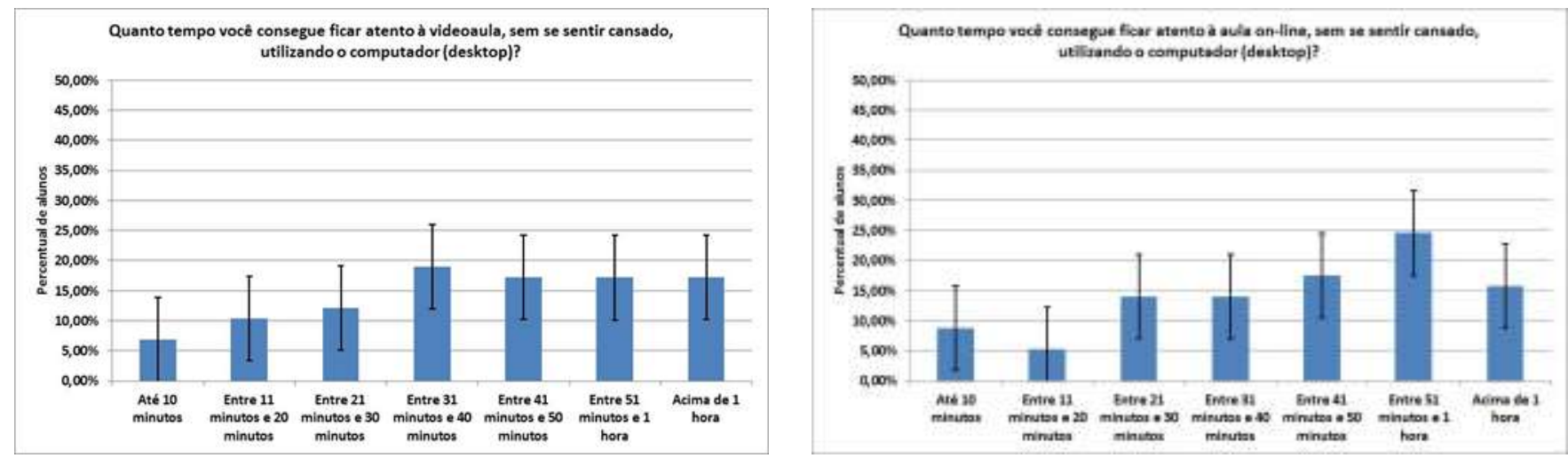

Fonte: Dos próprios autores.

Em tempos de pandemia onde em muitos lugares, e no IFES - Campus Itapina não foi diferente, a maioria dos alunos não estava habituada às novas formas de assistir às aulas. Na Figura 10 a ideia da pergunta era que, depois de um tempo já assistindo às aulas das metodologias de videoaulas e aulas online, qual foi a metodologia que mais agradou, mostrando que poderia ser viável a ser utilizada mesmo no retorno às aulas presenciais. A maioria dos alunos $(55,23 \%)$ escolheu o recurso de videoaulas, enquanto $44,77 \%$ acharam as aulas online mais interessantes. Este resultado provavelmente se encaixa dentro da 
perspectiva da facilidade de poder encontrar com certa facilidade um conteúdo em uma videoaula quando necessária, através das ferramentas de navegação existentes dentro das plataformas de vídeos, onde o discente pode avançar, retroceder, pausar, entre outros recursos. Além disso, a facilidade que a videoaula tem de poder assistí-la a hora que quiser, de acordo com o planejamento do aluno.

Figura 10 - Metodologia que mais agradou os alunos durante o período letivo 2020/2021.

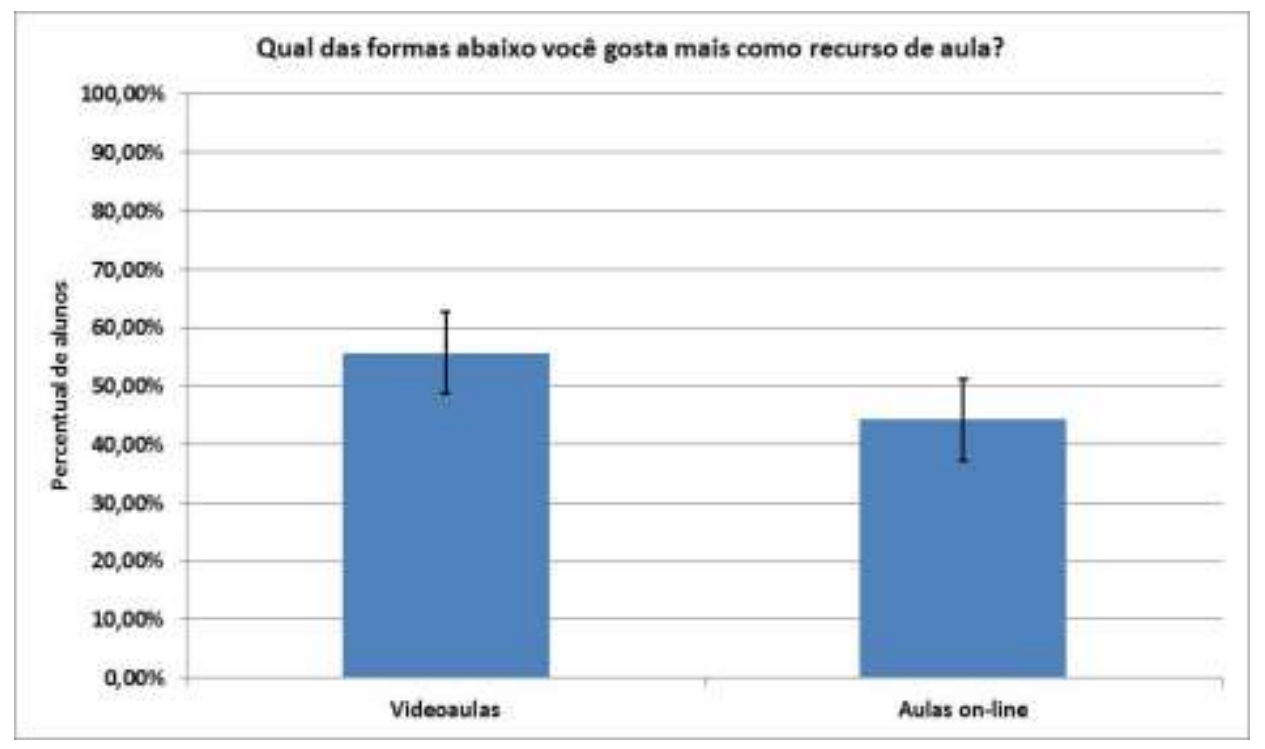

Fonte: Dos próprios autores.

\section{Considerações Finais}

O momento atravessado pela educação durante a pandemia mostrou deficiências do sistema educacional brasileiro, em que muitos professores não estavam aptos a lidar com as TDICs, onde também, muitos discentes viram como é o estudar através de uma tela durante longos períodos de tempo. Uma das características dessa geração $\mathrm{C}$, também chamada de geração Youtube pelo Google, é a adoção de vídeos como recurso para consumo de conteúdo. É visto que cada vez mais o número de smartphones e notebooks têm aumentado e que a adoção destes dispositivos no acesso de conteúdos para estudos é um nicho de mercado em crescente expansão e que a educação deve seguir essa tendência.

Os resultados mostrados neste trabalho, especificamente no que tange à experiência do aluno em relação ao uso dos dispositivos e tolerância no acompanhamento de aulas através da tela do smartphone e notebooks, deixam claro que o tamanho da tela é proporcional ao tempo de tolerância suportada pelo discente. Somado a isso, percebe-se claramente uma tendência da Geração C de ver vídeos cada vez mais curtos, objetivos e que sejam "diretos ao ponto". Ferramentas como o Reels do Instagram, a rede social TikTok e o recém criado Youtube Shorts, mostram uma tendência dessa geração no foco em conteúdos de curta duração e que vão de encontro ao que buscam. Portanto, independente da modalidade de ensino, seja presencial ou não, o planejamento curricular deve considerar a adoção e como usar esses dispositivos na relação ensino e aprendizagem. E por isso, uma abordagem metodológica que está começando a chamar a atenção da academia e possibilidade de aplicação na educação, e que tem foco nessa característica de transmissão de conteúdo de curta duração é o microlearning (Giurgiu, 2017).

Como trabalhos futuros serão realizados experimentos utilizando da metodologia de microlearning junto aos alunos de diferentes níveis de escolaridade (Ensino Médio e Superior) para análise da experiência do aluno em relação à metodologia. 


\section{Referências}

Artacho, E., Martínez, T., Martín, L., Marín, J., \& García, G. (2020). Teacher training and lifelong learning - The importance of digital competence in the encouragement of teaching innovation. Sustainability, 12,2852

Atmojo, A. E. P., \& Nugroho, A. (2020). EFL classes must go online! Teaching activities and challenges during COVID-19 pandemic in Indonesia. Register Journal, 13(1), 49-76.

Bailenson, J. N. (2021). Nonverbal Overload: A Theoretical Argument for the Causes of Zoom Fatigue. Technology, Mind, and Behavior, 2(1)

Cucinotta, D., \& Vanelli, M. (2020). WHO declares COVID-19 a pandemic. Acta Bio Medica: Atenei Parmensis, $91(1), 157$.

da Silva, J. F., \& Pinto, A. (2009, October). Geração C: Conectados em novos modelos de aprendizagem. In VIII Brazilian Symposium on Games and Digital Entertainment Rio de Janeiro, RJ-Brazil.

Doring, T., Cruz, E. P. F., \& Ribeiro, F. N. (2021). As tecnologias digitais de informação e comunicação priorizadas no contexto do ensino remoto emergencial no IFES-Campus Itapina: Digital information and communication technologies priorized in the context of emergency remote education at IFESCampus Itapina. Revista Cocar, 15(32).

Gil, A. C. (2002). Como elaborar projetos de pesquisa (Vol. 4, p. 175): Atlas.

Giurgiu, L. (2017). Microlearning an evolving elearning trend. Scientific Bulletin-Nicolae Balcescu Land Forces Academy, 22(1), 18-23.

Google Brasil (2012). Apresentação da Geração C: a geração youtube. https://think.storage.googleapis.com/intl/ALL_br/docs/introducing-gen-c-the-youtubegeneration_research-studies.pdf

Hundekari, J., Sisodiya, R., \& Kot, L. (2021). Smartphone Vision Syndrome Associated with Prolonged Use of Digital Screen for Attending Online Classes during COVID-19 Pandemic among Medical Students: A Cross-sectional Study. Journal of Clinical \& Diagnostic Research, 15(1).

Intolo, P., Shalokhon, B., Wongwech, G., Wisiasut, P., Nanthavanij, S., \& Baxter, D.G. (2019). Analysis of neck and shoulder postures, andmuscle activities relative to perceived pain during laptop computer use at a low-height table, sofa and bed. Work, 63(3), 361-367.

Joye, C. R., Moreira, M. M., \& Rocha, S. S. D. (2020). Distance Education or Emergency Remote Educational Activity: in search of the missing link of school education in times of COVID-19. Research, Society and Development, 9(7), 1-29.

Klapproth, F., Federkeil, L., Heinschke, F., \& Jungmann, T. (2020). Teachers' Experiences of Stress and Their Coping Strategies during COVID-19 Induced Distance Teaching. Journal of Pedagogical Research, 4(4), 444-452.

Lee, J. (2020). A neuropsychological exploration of Zoom fatigue. Psychiatric Times

Leeb, R.T., Bitsko, R. H., Radhakrishnan,L., Martinez, P., Njai, R., \& Holland, K.M. (2020). Mental health-related emergency department visits among children aged <18 years during the COVID-19 pandemic-Enited States, January 1-October 17, 2020. Morbidity and Mortality Weekly Report (MMWR), 69(45),1675-1680

Li, X., Zai, J., Zhao, Q., Nie, Q., Li, Y., Foley, B. T., \& Chaillon, A. (2020). Evolutionary history, potential intermediate animal host, and cross-species analyses of SARS-CoV-2. Journal of medical virology, 92(6), 602-611.

Liang, W., Guan, W., Chen, R., Wang, W., Li, J., Xu, K., \& He, J. (2020). Cancer patients in SARS-CoV-2 infection: a nationwide analysis in China. The lancet oncology, 21(3), 335-337.

Maniar, N., Bennett, E., Hand, S., \& Allan, G. (2008). The effect of mobile phone screen size on video based learning. J. Softw., 3(4), 51-61.

McGinty, E. E., Presskreischer, R., Anderson, K. E., Han, H., \& Barry, C. L. (2020). Psychological distress and COVID-19-related stressors reported in a longitudinal cohort of US adults in April and July 2020.JAMA, 324(24),2555-2557

Moreira, J. A., \& Schlemmer, E. (2020). Por um novo conceito e paradigma de educação digital onlife. Revista uFG, 20(26).

Nascimento, M. C., Mansur, G. S. S., \& Gomes, G. R. R. (2020). Pandemia, Tecnologias Digitais E Educação: Pensamentos Sobre A Escola De Hoje [Ebook]. In Pandemia E Suas Interfaces No Ensino (pp. 95-108). Pedro e João Editores.

Niemi, H. M., \& Kousa, P. (2020). A case study of students' and teachers' perceptions in a Finnish high school during the COVID pandemic. International journal of technology in education and science.

Peper, E., Harvey, R., \& Faass, N. (2020). TechStress. How technology is hijacking our lives, strategies for coping,and pragmatic ergonomics. Berkeley, CA: North Atlantic Books

Peper, E. (2021). "Resolve eyestrain and screen fatigue.” Well Being Journal,.30, Winter 2021

Peper, E., Wilson, V., Martin, M., Rosegard, E., \& Harvey, R. (2021). Avoid Zoom fatigue, be present and learn. NeuroRegulation, 8(1), 47-47.

Popescu, E. F., Tătucu, M., \& Dobromirescu, V. (2021). Students' Well-being in Online Education in Covid-19 Context. International Journal of Education and Research, 9(2).

Purwanto, A, Pramono, R, Asbari, M, Hyun, C. C, Wijayanti, L. M, \& Putri, R, S. (2020). Studi Eksploratif Dampak Pandemi COVID-19 terhadap Proses Pembelajaran Online di Sekolah Dasar. EduPsyCouns: Journal of Education, Psychology and Counseling, 2(1), 1-12 
Research, Society and Development, v. 10, n. 12, e228101220436, 2021

(CC BY 4.0) | ISSN 2525-3409 | DOI: http://dx.doi.org/10.33448/rsd-v10i12.20436

Spataro, J. (2020, November 9). The future of work-the good, the challenging \& the unknown. Microsoft 365 Blog. https://www.microsoft.com/enus/microsoft-365/blog/2020/07/08/future-work-good-challenging-unknown/

Werneck, G. L., \& Carvalho, M. S. (2020a). A pandemia de COVID-19 no Brasil: Crônica de uma crise sanitária anunciada. Cadernos de Saúde Pública, 36(5), e00068820. https://doi.org/10.1590/0102-311x00068820 